\title{
Fiction Popularity Prediction Based on Emotion Analysis
}

\author{
Xing Wang \\ ITMO University \\ Saint Petersburg, Russia \\ w4ngxing@gmail.com
}

\author{
Shouhua Zhang \\ University of Oulu \\ Oulu, Finland \\ shouhua.zhang@oulu.fi
}

\author{
Ivan Smetannikov \\ Machine Learning lab \\ ITMO University \\ Saint Petersburg, Russia \\ ismetannikov@itmo.ru
}

\begin{abstract}
In addition to bringing us knowledge, books also bring us emotional experiences. How do the emotional fluctuations brought by books affect readers' evaluation of them? What is the difference in emotional fluctuations between books of different popularity? In this paper, we model and analyse the emotional fluctuations of different fiction books with different popularity and study the feasibility of predicting the popularity of fiction books using emotional fluctuations and recurrent neural networks. A new dataset is also generated to support this research and other related researches. Our proposed method obtained the best accuracy of $73.4 \%$ for predicting the popularity of fiction books and $41.4 \%$ for predicting genres. Some interesting data insights are also extracted from the dataset.
\end{abstract}

\section{CCS CONCEPTS}

- Computing methodologies $\rightarrow$ Artificial intelligence $\rightarrow$ Natural language processing $\rightarrow$ Natural language generation $\bullet$ Computer methodologies $\rightarrow$ Machine learning $\rightarrow$ Machine learning approaches $\rightarrow$ Neural networks $\bullet$ Computer methodologies $\rightarrow$ Machine learning $\rightarrow$ Learning paradigms $\rightarrow$ Supervised learning

\section{KEYWORDS}

Popularity Prediction, Emotion Analysis, Natural Language Processing.

\section{Introduction}

People love to read books for relaxing, especially fiction stories in their free time. And all the fiction books we read reflect a variety of different emotions that lead us into different moods as the plot progresses. Something like a happy-ending love story makes us feel happy and optimistic while some tragic stories make us feel sad and blue. In addition, our feelings keep changing as we witnessed the joys and sorrows of the characters in the fiction story. To some extent, when we say we are reading and enjoying a certain fiction book, we are actually enjoying the strong feelings and the process of emotional changes it brings us. How do the emotional features of a book affect its potential popularity? There are researchers have conducted interesting and valuable related researches.

Reagan [1] later used a series of techniques including matrix decomposition by singular value decomposition (SVD), unsupervised learning and supervised learning to study the emotional arcs of fiction books from Project Gutenberg ${ }^{1}$. Result of experiments shows that different complex emotional trajectories of different books can be formed from a combination of the same six basic emotional arcs. They also studied the potential relationship between emotional arcs and downloads of books and found that certain types of emotional arcs have a greater number of downloads, which indicates that books containing these types of emotional arcs are more successful in a sense.

Later in 2018, Maharjan [2] performed a series of tasks to model the emotion flows in different types of books and studied the correlation between the emotion flow of a book and its potential success measured by the average rating. This paper divided all the books into two groups (successful and unsuccessful) based on the average ratings of books from Goodreads ${ }^{2}$, then made use of emotion lexicon to build emotion flows of books. They performed data analysis tasks upon the generated emotion flows and found that emotion flows in a ups and downs shape contains more successful books than other shapes of emotion flows. And it revealed the findings of earlier researches that different types of books have different emotional compositions. The proposed model which is a combination of gated recurrent network (GRU) Cho [3] with attention mechanism yields the best f1 score of 0.674 while predicting the success of books.

Except from methods of predicting book's success based on the content, there are also researches that studied the impact of features that have no relation with the content on the book's success. In 2019, Wang [4] studied the relationship be-tween the sales of books and features other than content of books. They studied the three feature groups: publisher features, author features (author's visibility, author's sales history, etc.) and features related to the book (general information, book's sales history, topic information, etc.) applying a series of machine learning methods such as KNN and linear regression. After conducting several experiments on a dataset that consists of 170,927 books from NPD Bookscan ${ }^{3}$, this study found

\footnotetext{
${ }^{1}$ https://www.gutenberg.org/wiki/Main_Page

${ }^{2}$ https://www.goodreads.com/
} 
that for fiction books, regardless of the content of a book, the factor that impacts the sales of a book most is the author's previous sales.

\section{Methodology}

In this research, we first model the emotional information of books using our proposed emotion trace. Emotion trace is a phrase used in this article to represent the emotion aware vector of a fiction book generated by the proposed method based on the full content of book and the NRC Emotion Intensity Lexicon ${ }^{4}$. After the generation of emotion traces these generated emotion aware vectors along with their labels measuring the potential popularity which is based on readers' real ratings of books will be fed into a GRU based deep learning network. This model consists of two layers of gated recurrent unit connected by a dropout layer and before the last softmax classifier layer an attention layer is applied upon the sequences produced by the previous GRU layers. After training, the trained model can be used to predict the popularity of books based on the content.

\subsection{Generation of Emotion Trace}

The generation of emotion trace is the most fundamental and critical part of this research because subsequent emotion analysis and popularity prediction tasks rely on the generated emotion trace and the quality of generated emotion trace has a significant impact on the quality of data insights that we can extract from the emotion trace later and the performance of popularity prediction tasks. When considering the construction of the emotion trace, it's important to keep in mind that the emotion trace should also be able to represent the fluctuations of various emotions in an entire book. Also, since the emotion trace will be used to train several models based on machine learning and deep learning techniques, it should be somewhat normalized so that the training process is smoother. To meet all the mentioned requirements, an efficient way to extract emotion information from text is essential. There are several usable methods to extract sentiment and emotion information from text. Some of them extract emotion automatically using machine learning approaches and some of them rely on emotion lexicons just as mentioned earlier in the introduction section. After investigation and comparison of different text emotion extraction methods, we decided to use emotion lexicons directly since there are already very good emotion lexicons out there which are proven to be very useful in many tasks. Among several representative sentiment/emotion lexicons, the NRC Emotion Intensity Lexicon is selected as the labels of the lexicon can meet the requirements of the emotion trace generation and it has been proven to work well with emotion analysis tasks of books [2]

\subsection{The NRC Emotion Intensity Lexicon}

The lexicon is developed by the researchers from National Research Council Canada and it is a part of the larger NRC Sentiment and Emotion Lexicons that consists of a variety of different lexicons for different application purposes [5, 6]. These different lexicons provide the possibility to analyze the sentiment and emotions of different type of texts and in different ways. For example, the hashtag related lexicons can be used to analyze the sentiment polarity and emotions of hashtags in text. The word-color association maps words to different colors based on their emotion orientations. In this research we only use the NRC Emotion Intensity Lexicon which associates words with specific emotions to extract the emotion information from books as there are very few hashtags in books and all the punctuation should be removed before the emotion trace generation. The NRC Emotion Intensity Lexicon provides word level emotion associations which map words to different types of emotions (there are eight emotions in total: anger, disgust, fear, sadness, anticipation, joy, trust and surprise) and the intensity values. The range of the intensity value of emotions is $(0$, 1) and the volume of the English corpus is about 10,000 and it also provides the automatic translations that are available in 40 different languages.

\subsection{Emotion trace}

Since our emotion trace should reflect the emotion fluctuations through the whole book, we first divide the content of books into a number of equal sized chunks by the number of words. We regard each chunk of text as a time point at which we measure the distribution of all the specific emotions. Then we concatenate the emotion information at all the time points and get the emotion fluctuation representation of a book. The number of chunks can be selected freely from 1 to 100 considering the different length of books. If only one chunk is used it means that we don't split the content of books at all and the emotion trace is generated at just one time point (the whole content of book), so it just represents the overall distribution of different emotions in the book. We setup experiments in this way because we want to study the impact of the number of time points on the performance of prediction tasks.

To generate the emotion trace for each time point we first initialize an eight-dimensional emotion vector for each time point, where each dimension corresponds to one emotion in the emotion lexicon. At a time point we search each word in the chunk in the NRC Emotion Intensity Lexicon. If there is a match of any emotion in the initialized emotion vector, we increase the value of the corresponding dimension by the intensity value of the matched emotion. After all words in the chunk are searched, we have an eight-dimensional vector representing the distribution of various emotions in this chunk. However, considering that the length of books often differ in a dataset and this may interfere with the comparison of emotional changes between different books, and it is also not conducive to the training of our machine learning and deep learning based models because some emotion values could be very large in some books and very small in some other books, it is necessary to find a way to normalize the emotion vector we generated to avoid the mentioned problems. A simple method that is also used in this study is to calculate the proportion of each emotion value in the vector to the sum of all emotion values. In this way, we get an emotion vector in which the value of each dimension represents the proportion of the corresponding emotion

\footnotetext{
${ }^{4} \mathrm{http} / / /$ sentiment.nrc.ca/lexicons-for-research/
} 
in the chunk and each value in the emotion vector is between 0 and 1. The generation of the emotion trace of a chunk can be shown as a schematic diagram as follows:

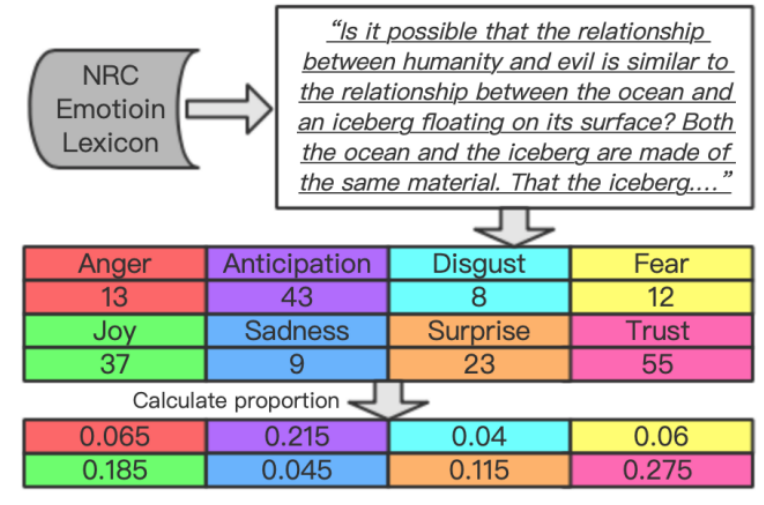

Figure 1: Demonstration on how to generate the emotion vector for a piece of text (The text in the figure is quoted from The Three-Body Problem 5 by Liu Cixin)

\subsection{Representation and Input}

After the generation of emotion trace of each chunk, we simply combine all the emotion vectors that hold the emotional proportion at each time point from all the chunks and make them be our emotion aware matrix. And at this phase our emotion trace of a book is generated. The emotional proportions of each chunk in the emotion trace stand for the emotional fluctuations of all the eight different emotions through all the time points in the book. In this way, the emotional changes of a book from beginning to end is modeled. The number of time points is selected by researchers freely when conducting experiments and it depends on your choice of granularity of emotion analysis. In this study, we performed tasks to experiment with different number of chunks.

Then the generated emotion traces of a collection of fiction books will be used to perform emotion analysis tasks to get some meaningful data insights and fed into several machine learning and deep learning models for training. So, the format of the emotion trace representation should be easy to fit in various of models with different requirements of input shape with minor modifications. Let $N$ be the number of chunks of each book (the number of chunks could be 1 in the case that we only want to calculate the overall proportion of various emotions in the book), the format of emotion trace of each book $x_{i}$ could be represented by the equation below:

$$
x_{i}=\left[\left[\frac{V_{j}}{\sum_{j=1}^{E} V_{j}} ; \ldots\right]_{E} ; \ldots\right]_{N}
$$

Where $E$ is the number of emotion types which is 8 in our case, $V_{j}$ is the emotion value of the $j$-th emotion in one chunk. So, for each book we have an emotion trace consists of $N$ emotion vectors that has a dimension of $E$. With this representation of emotion trace, the emotional changes of a book can be easily visualized. And it's easy to adjust the shape to fit in different models. For example, we can regard each vector in the emotion trace as the input at each time step when we want to train a RNN based network [7] using the emotion trace data, and we can simply flatten the emotion trace matrix into a vector if we want to fit our data in a linear model.

\subsection{Model}

Since our generated emotion trace represents the fluctuation of different emotions through the book, in the stage of considering the appropriate model, we want the model to make full use of the sequential information about the emotional changes of books. After investigation and comparison of some models in both machine learning and deep learning areas, RNN models stand out as the ideal models for this study as RNN networks are very suitable for processing sequential data and the input shape of RNN models is a set of sequences so that each time step in a RNN network can exactly correspond to one emotion vector of a chunk in the emotion trace. By this way, our emotion trace can benefit from the nature of time series processing as the RNN models have the ability to "remember" previous states while training the following series of data so that the emotional fluctuation information can be well learned by the model and applied to subsequent prediction tasks. There are several representative networks that are in the RNN family such as the vanilla RNN network, the long short-term memory (LSTM) [8, 9] network and gated recurrent unit (GRU) network, etc. These networks all have the ability to process time series data. However, an original RNN network simply "remembers" the latest previous states while training so that the relationship between the earlier states and the current memory is lost. Also, it may have the problem of vanishing gradient and exploding gradient [8, 9]. Using LSTM networks may avoid such shortcomings and problems as it introduces a separate cell state to save the long-term memory and the gate mechanism is responsible for filtering information to achieve the control of long-term memory. GRU networks can be regarded as a variant of LSTM networks as it benefits from some simplifications and optimizations while having the same advantages of LSTM networks. Since experiments the GRU based network yields the best result, it is used as the proposed model in this study to predict the fiction popularity.

As shown in the figure 2, our proposed model consists of two GRU layers. The second GRU layer is followed by an attention layer. Attention mechanism is applied in our proposed model as the attention mechanism allows the model to focus on important information and fully learn and absorb it while learning [10]. After the attention layer there is a softmax dense layer which classify books into different groups of popularity degrees or genres.

Once a book's emotion trace is generated based on the NRC Emotion Intensity Lexicon, then emotion vector will be fed into the first layer. Given the fact that GRU network needs its input to be in a 3D vector shape (num_samples, time_steps, feature_dim), our emotion trace representation can be easily fed into the model as it meets the input requirement by letting our $N$ emotion vectors correspond to $N$ time_steps in the GRU network (each chunk stands for one time_step). And the feature_dim should be naturally set as

\footnotetext{
${ }^{5}$ https://en.wikipedia.org/wiki/The_Three-Body_Problem_(novel)
} 
the dimension of the chunk emotion vector $(\mathrm{E})$ which is eight in our case. And we use categorical cross entropy as the loss function and adam as the optimizer of our model to perform classification task (predicting popularity degrees). And parameters such as dropout rate and learning rate are also tuned through multiple trials. Early stopping criteria is also applied in the stage of training to prevent overfitting.

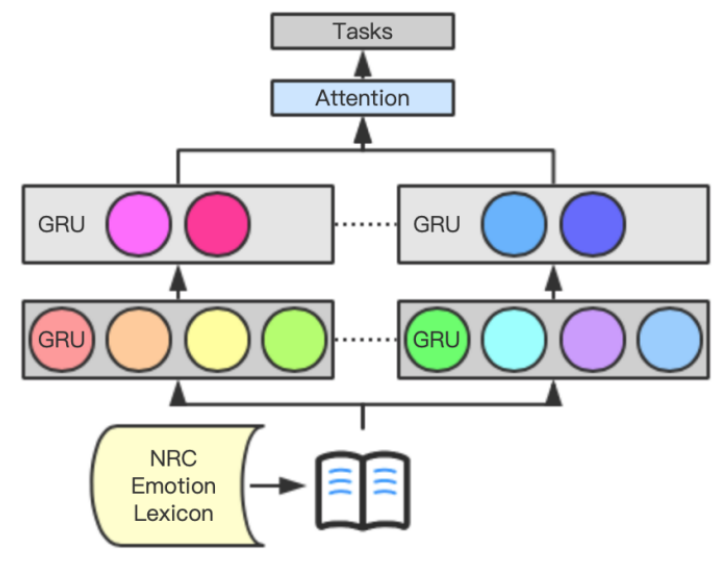

Figure 2: Architecture of the proposed model

\section{Experiments}

\subsection{Dataset}

Since we want to focus on fiction books and datasets that already exist either contain too broad types of books or have no specific rating information, we had to collect and generate dataset ${ }^{6}$ on our own. We scraped our content of books from Project Gutenberg and ratings as well as number of ratings from Goodreads. After data filtering and cleaning, we saved about 1000 fiction books from 10 different genres (Science Fiction, Children's Fiction, Historical Fiction, Humor, Fantasy, Western, Detective Fiction, School Stories, Movie Books, Horror) and the number of books in each genre is in a range of 50 to 200.

After filtering our books, we need to generate data labels. We generate a discrete popularity degree (range from 1 to 5) for each book based on its rating value. The rule of generating popularity degrees from ratings in this study is to classify each book into different degrees of popularity according to the different numerical ranges of the rating. Specifically, a book has a degree of popularity of 1 if the rating is between 0 and 1.5, 2 if the rating is between 1.5 and 2.5, 3 if the rating is between 2.5 and 3.5, 4 if the rating is between 3.5 and 4.5 and 5 if the rating is between 4.5 and 5 . The reason to generate popularity degrees is that predicting the approximate popularity range of a book is more practical as predicting an accurate rating value would be too difficult and not actually practical in real usage scenarios. Currently in this study, only rating value is considered when generating popularity degrees.

\footnotetext{
${ }^{6}$ Generated dataset can be found here: https://github.com/wangx 1ng/UnifiedEmotion-Trace
}

However, other properties such as the number of rating and number of reviews of a book can be also added as one of the factors that model the popularity degrees as these properties indicate how actively a book is discussed. After generating popularity labels, we clean the content books by cutting the Project Gutenberg statement in the text and lowercase all the words so it can be matched by the words in emotion lexicon which only contains words in lowercase. At this point, our dataset is generated.

In summary, we crawled data from the Internet and got a dataset that consists of the content of about 1000 fiction books in 10 different genres labeled by the genre and popularity degrees ${ }^{7}$.

\subsection{Experiment Setup}

After introducing our proposed method to model the emotion fluctuation in fiction books and the architecture of the model that we proposed to train the generated emotion trace to predict the fiction popularity degrees as well as genres of books. We are ready to conduct the experiments and perform the classification tasks to check the performance of the proposed method. We set up two groups of experiments in which one experiment group is about the comparison of our proposed method and the method by Maharjan [2] and the other one is conducted on our newly generated dataset aiming to prove the feasibility of predicting fiction popularity degrees based on the emotional fluctuation of books.

For the comparison experiments, we used exactly the same dataset which was used in the paper mentioned above that consists of books in 10 different categories labeled by the book success (654 successful and 349 unsuccessful). Based on this dataset we performed the same task of predicting the success of books using our method and proposed model. The result of this experiment shows that our proposed method has a higher $\mathrm{F} 1$ score in predicting book's success.

To study the feasibility of predicting degrees of popularity of fiction books using emotional fluctuation features, we also set up some baseline experiments apart from conducting experiment on the newly generated dataset using the proposed method. The baseline models we use to be compared with the proposed model are as follows:

SVM + Emotion trace: SVM has been proved to be efficient widely in many classification or regression tasks [11]. So, it's often used as a baseline model for many tasks. We just feed the emotion vector (emotion trace) generated by our propose method into the model when training this network. We concatenate our $N$ emotion vectors in dimension of $E$ into a one-dimensional vector as SVM is not able to handle sequential input.

Random Forest + Emotion trace: random forest also known as random decision forest is also an efficient model in both classification and regression tasks [12]. We also use our generated emotion vector as the input of this model. Just like what we did with SVM, the input of this model is also the concatenation of the emotion vectors of all the chunks.

\footnotetext{
${ }^{7}$ Code for dataset crawling can be found here: https://github.com/wangx1ng/Gutenberg-Collector
} 
LSTM + Emotion trace: as a variant of recurrent neural networks (RNNs), using LSTM combined with the emotion trace generated by our proposed method is a good setup to prove whether the emotional fluctuation modeled by our emotion trace help the prediction or not as the previous two baseline models don't have the ability to learn sequential information from the input data. By comparing the performance of them we can know if our emotion trace helps the task. The input of a LSTM network is just like our proposed model, so the number of chunks equals to the number of time steps of the model and one emotion vector of a chunk is processed at each time step.

LSTM + Keras embedding: Instead of feeding the model with our generated emotion trace, in this setup we set the first layer of this network as a Keras Embedding layer so that the embedding generated by the Keras tokenism and sequence APIs will be fed as the input sequence. In this way we can verify the effectiveness of the emotion trace generated by our method by comparing the result with the result of the previous baseline.

As for the train test splitting, for both our proposed model and baseline models, we split the dataset randomly into two groups in which there are $30 \%$ of books in the testing set and $70 \%$ of books in the training set.

\section{Results}

As table 1 shows, our proposed method and model have a better $\mathrm{f} 1$ score while performing the same success prediction task on the same dataset that Maharjan [2] used which proves that our proposed method models emotional fluctuation of books better. One possible reason is that the proportion of each emotion is calculated instead of just calculating the average of each chunk in our method so that it eliminates the effect of the different lengths of books in the dataset. If the lengths of books in the dataset differ too much, the difference of emotion vector values between different books would be too large as well so that it doesn't accurately reflect the emotional fluctuations of all books. But in our method, we use the relative emotion proportion to represent the emotion status at each time point, so it's fairer when modeling the emotional fluctuation of two books of different lengths or analyzing them in a same context.

Table 1: Results of the comparison task (evaluated by f1 score)

\begin{tabular}{|l|l|}
\hline Methods & Best F1 Score \\
\hline NRC + SVM & 0.611 \\
\hline $\begin{array}{l}\text { Emotion } \\
\text { Flow }\end{array}$ & 0.674 \\
\hline Ours & 0.698 \\
\hline
\end{tabular}

Another possible reason could be that we discard the sentiment polarity (positive and negative) in our method as many words in the NRC Emotion Lexicon (used by Maharjan [2]) have both emotion association and sentiment polarity at the same time so that the sentiment polarity could be somewhat redundant and interfere the process of emotional fluctuation modeling.

\subsection{Results of Popularity Prediction}

The results show (in the table 2) that our method give decent accuracy score for both popularity prediction task and genre prediction task which indicates that our method model the emotional fluctuation of fiction books effectively and it is feasible to predict the popularity of fiction books based on the emotional features extracted from the content. Our proposed model outperforms the rest baseline models as both the best accuracy score of popularity prediction (0.734) and the best accuracy score of genre prediction $(0.414)$ are obtained by our proposed model.

If we analyze the results in more detail, we can discover more things. As table 2 and figures 3-5 show, our method achieved the best accuracy in predicting popularity degrees when books are divided into 20 chunks and the accuracy score starts to decrease when the number chunks increases to 30 . We can empirically conclude that for our dataset, the appropriate granularity to model the emotional fluctuation is to divide the book into 20 equal chunks.

However, the choice of number of chunks could be somewhat related to the length of books. Also, the fact that the result obtained from emotion trace generated by splitting books into chunks is better than the results obtained from emotion trace generated by not splitting the book proves that modeling the emotional fluctuation by measure the emotion distribution at different time point of a book is helpful.

Table 2: Results of popularity and genre prediction

\begin{tabular}{|l|l|l|l|}
\hline Methods & Chunks & $\begin{array}{l}\text { Accuracy } \\
\text { (popularity) }\end{array}$ & $\begin{array}{l}\text { Accuracy } \\
\text { (genre) }\end{array}$ \\
\hline LSTM+Keras Embeding & - & 0.576 & 0.291 \\
\hline SVM+Emotion Trace & 1 & 0.730 & 0.342 \\
\hline SVM+Emotion Trace & 10 & 0.730 & 0.327 \\
\hline SVM+Emotion Trace & 20 & 0.730 & 0.317 \\
\hline SVM+Emotion Trace & 30 & 0.730 & 0.327 \\
\hline RF+Emotion Trace & 1 & 0.730 & 0.302 \\
\hline RF+Emotion Trace & 10 & 0.730 & 0.281 \\
\hline RF+Emotion Trace & 20 & 0.730 & 0.291 \\
\hline RF+Emotion Trace & 30 & 0.730 & 0.295 \\
\hline LSTM+Emotion Trace & 1 & 0.730 & 0.302 \\
\hline LSTM+Emotion Trace & 10 & 0.730 & 0.410 \\
\hline LSTM+Emotion Trace & 20 & 0.730 & 0.388 \\
\hline LSTM+Emotion Trace & 30 & 0.730 & 0.396 \\
\hline Ours & 1 & 0.730 & 0.414 \\
\hline Ours & 10 & 0.730 & 0.406 \\
\hline Ours & 20 & 0.734 & 0.353 \\
\hline Ours & 30 & 0.716 & 0.367 \\
\hline
\end{tabular}

Unlike the popularity prediction, the best accuracy in predicting genres is obtained when the number of chunks is 1 which means books are not divided at all and the emotion vector calculated in 
X. Wang et al.

this way actually represents the overall emotion distribution of a book. This may indicate that we might distinguish different genres of fiction books better by just using the overall emotion distribution.

And by comparing the results obtained by LSTM with Keras embedding input and LSTM with our generated emotion trace, we can observe a significant performance improvement in both popularity prediction and genre prediction. This is a strong proof of the effectiveness of our method as these two setups have the same model architecture but different input vectors.

\subsection{Data insights}

By analyzing the emotion trace generated by the proposed method, some interesting data insights regarding the emotion patterns of different genres of fiction books are found. Since the best accuracy score is obtained at 20 chunks, we divided all the books into 1-20 equal chunks and made some visualizations of books' emotion traces.
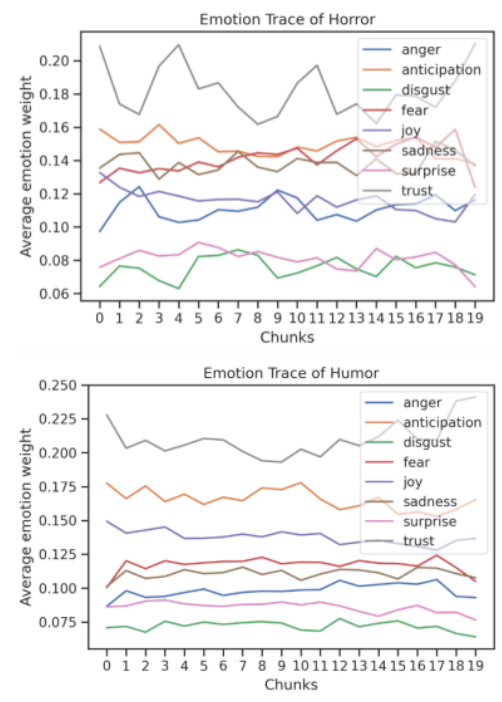

Figure 3: Emotion traces of books in Horror and Humor

Although the emotions that account for the largest proportion of the whole are trust and anticipation for almost all genres of books, we can still observe from the two figures above that there is a significant difference regarding emotions like joy and fear between horror books and humor books. The average proportion of fear and joy is about $14 \%$ and $12 \%$ respectively in horror books while the they are about $12 \%$ and $14 \%$ in humor books. This result confirms our intuitive impression that we get more joyful feelings when reading humor books and more feelings of fear when reading horror books.
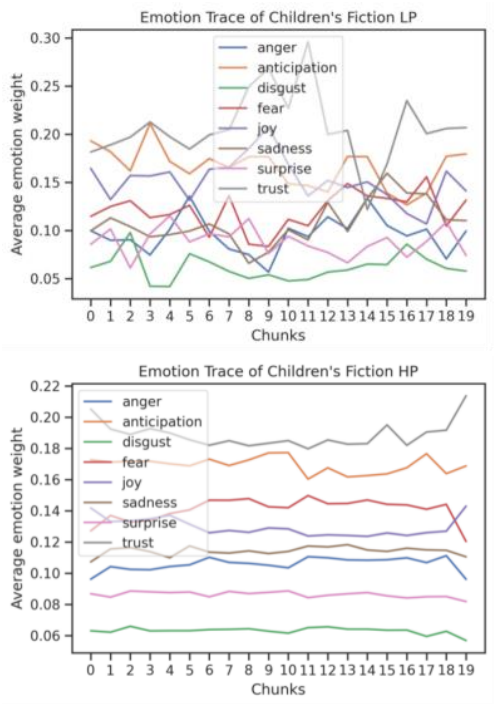

Figure 4: Emotion traces of Children's Fiction in low and high popularity groups

After visualizing the emotion of children's fiction books of both low popularity group (popularity is equal or less than 2) and high popularity group (popularity is equal or larger than 4), we observed that there is a big difference between the emotion shape of books from these two groups. We can clearly observe that the emotional fluctuation is very strong in the low popularity group while all emotions are very stable through the books in the high popularity group. This means that children do not really like emotional roller coasters which proves the intuitive impression that children really enjoy cute stories with warm and positive emotions and not so strong emotional fluctuations because of their young age. But apparently this is not the case for adults!

By visualizing the emotion traces of detective fiction books in both the low popularity group and the high popularity group, we observed the opposite result that emotional fluctuations are strong in detective fiction books from high popularity group while emotions are stable in those books from low popularity group. We can speculate from such results that the adult readers of detective fiction like the emotional roller coaster with the criminal investigation in the plot.

Similar findings regarding the different emotional fluctuation between low popularity books and high popularity books can be also found in fiction books of some other genres. 

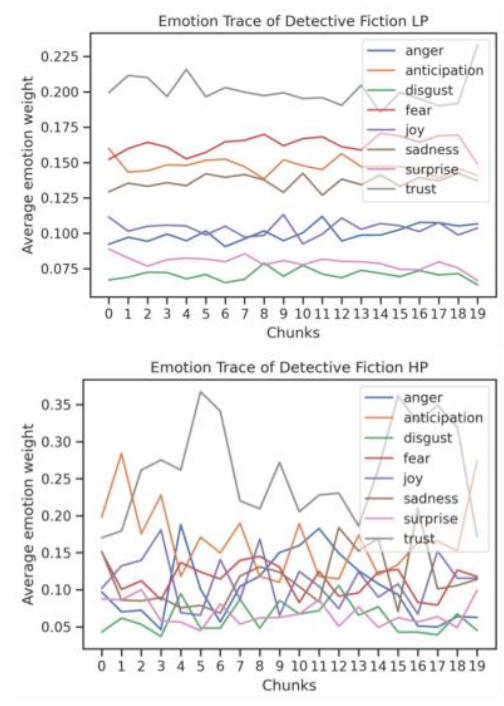

Figure 5: Emotion traces of Detective Fiction in low and high popularity groups

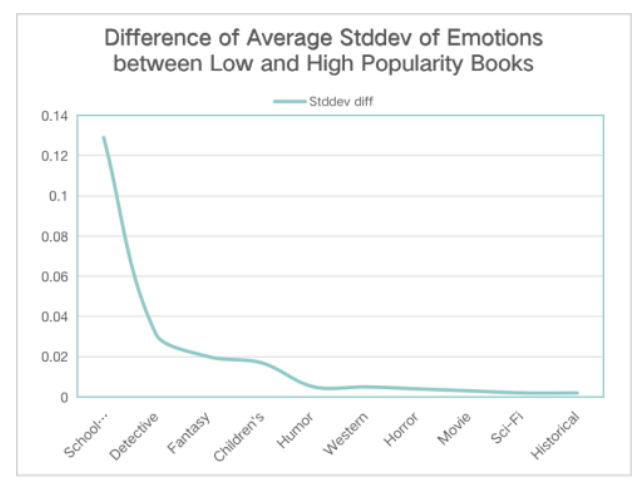

Figure 6: Difference of average standard deviation of emotions between books in low and high popularity groups

As shown in the figure 6 , we visualized the difference of standard deviation between books in low and high popularity groups from each genre. And we found that the similar phenomenon that the emotional fluctuation between books from low and high popularity groups differs a lot that happens with children's fiction and detective fiction also happens with books in school stories and fantasy fiction. Both for school stories and fantasy fiction, the average standard deviation of emotions in the low popularity group is less than in the high popularity group. The reason why it also happens with school stories could be the same as the reason with children's fiction as the main readers of school stories are children as well so that they have similar tastes. And the average difference of standard deviation of emotions in science fiction is very little which indicates that the emotional fluctuation doesn't differ much in science fiction and books in low and high popularity groups. In some way, this fits our intuitive impression that for science fiction, it is more of an awesome idea than a wave of emotions that determines whether people like it or not. Similar findings are found in historical fiction which is also a serious genre that has a target audience of hardcore readers.

\section{Conclusions and Future work}

In this research, we studied the feasibility of predicting popularity of fiction books using features like emotional fluctuation by machine learning and deep learning techniques. To support this research, a new dataset which consists of the content of fiction books from ten different genres and their popularity degrees was created and based on which a series of experiments are conducted to prove our hypothesis. Some emotion analysis tasks based on our proposed method and our generated dataset were also performed to extract some interesting data insights. As the research has demonstrated, our proposed method obtained better f1 score according to the result of the comparative experiment which shows that our method is simple yet effective in comparison with the other method [2]. Also, the result of the experiments that were conducted based on our newly generated dataset shows that it improves the performance of models in both popularity prediction and genre prediction basks by modeling the emotional fluctuation using our method. Our proposed model obtained decent result in both popularity prediction and genre prediction tasks which proves the feasibility of using emotional features to predict the popularity of fiction books. The data insights derived from the emotion analysis tasks based on our generated dataset confirmed that the potential popularity and genre of fiction books are related to the emotional fluctuations to a certain extent. Having been proved to be useful, the generated dataset can be also used for further researches in this area.

\section{ACKNOWLEDGMENTS}

This research received valuable help from Shouhua Zhang of University of Oulu, Oulu, Finland. This work is financially supported by the Government of the Russian Federation, grant 0808 .

\section{REFERENCES}

[1] AJ Reagan et. al. (2016). The emotional arcs of stories are dominated by six basic shapes. EPJ Data Science, 5(1), 31.

[2] S. Maharjan et. al., 2018, Letting emotions flow: Success prediction by modeling the flow of emotions in books, arXiv:1805.09746.

[3] K. Cho et. Al, 2014, Learning phrase representations using RNN encoder-decoder for statistical machine translation, arXiv:1406.1078.

[4] X Wang et. al. (2019). Success in books: predicting book sales before publication. EPJ Data Science, 8(1), 31.

[5] S Mohammad (2011). From Once Upon a Time to Happily Ever After: Tracking Emotions in Novels and Fairy Tales. ACL HLT, 105.

[6] SM Mohammad and PD Turney (2013). Crowdsourcing a word-emotion association lexicon. Computational Intelligence, 29(3), 436-465.

[7] A. Sherstinsky, 2018. Fundamentals of recurrent neural network (rnn) and long short-term memory (lstm) network, arXiv:1808.03314.

[8] S. Hochreiter et. al., 2001, Gradient flow in recurrent nets: the difficulty of learning long-term dependencies, https://ml.jku.at/publications/older/ch7.pdf.

[9] S Hochreiter and J Schmidhuber (1997). Long short-term memory. Neural computation, 9(8), 1735-1780.

[10] D. Bahdanau, K. Cho and Y. Bengio, 2014, Neural machine translation by jointly learning to align and translate, arXiv:1409.0473.

[11] C Cortes and V Vapnik (1995). Support-vector networks. Machine learning, 20(3), 273-297.

[12] TK Ho (1995). Random decision forests. Proceedings of 3rd international conference on document analysis and recognition, 278-282. 